\title{
Globe
}

Revue internationale d'études québécoises

\section{Ce passé qui ne passe pas. La grande noirceur catholique dans les films Séraphin. Un homme et son péché, Le Survenant et Aurore}

\section{This Past that Does Not Pass. The Catholic Great Darkness in the Films Séraphin. Un homme et son péché, Le Survenant and Aurore}

\section{Éric Bédard}

Volume 11, numéro 1, 2008

La religion au Québec. Regards croisés sur une intrigue moderne

URI : https://id.erudit.org/iderudit/1000492ar

DOI : https://doi.org/10.7202/1000492ar

Aller au sommaire du numéro

Éditeur(s)

Globe, Revue internationale d'études québécoises

ISSN

1481-5869 (imprimé)

1923-8231 (numérique)

Découvrir la revue

Citer cet article

Bédard, É. (2008). Ce passé qui ne passe pas. La grande noirceur catholique dans les films Séraphin. Un homme et son péché, Le Survenant et Aurore. Globe, 11(1), 75-94. https://doi.org/10.7202/1000492ar
Résumé de l'article

Les films de genre sont généralement un excellent reflet des valeurs dominantes d'une époque. Le genre historique peut donc constituer un terrain d'investigation fort intéressant pour qui cherche à comprendre le rapport d'une époque à son passé. Les films Séraphin. Un homme et son péché (2003), Le Survenant (2005) et Aurore (2005), qui marquent un retour des " classiques " dans le cinéma québécois, ont été analysés dans cette perspective. Ces trois films témoignent d'un rapport trouble à l'héritage religieux, toujours associé à l'idée de "Grande noirceur ». L'expérience religieuse des Canadiens français y est travestie et le rôle de l'Église et de son clergé, jugé très sévèrement. d'utilisation que vous pouvez consulter en ligne. 


\title{
CE PASSÉ \\ QUI NE PASSE PAS. \\ LA GRANDE NOIRCEUR \\ CATHOLIQUE DANS LES \\ FILMS SERAPHIN. UN \\ HOMME ET SON PECCHÉ,LE SURVENANT ET AURORE
}

\author{
ÉRIC BÉDARD \\ Université du Québec à Montréal
}

$+++4++4+++4++4++4++4+4+$

Résumé - Les films de genre sont généralement un excellent reflet des valeurs dominantes d'une époque. Le gente historique peut donc constituer un terrain d'investigation fort intéressant pour qui cherche à comprendre le rapport d'une époque à son passé. Les films Séraphin. Un homme et son péché (2003), Le Survenant (2005) et Aurore (2005), qui marquent un retour des "classiques" dans le cinéma québécois, ont été analysés dans cette perspective. Ces trois films témoignent d'un rapport trouble à l'héritage religieux, toujours associé à l'idée de "Grande noirceur". L'expérience religieuse des Canadiens français y est travestie et le rôle de l'Église et de son clergé, jugé très sévèrement.

\section{This Past that Does Not Pass. The Catholic Great Darkness in the Films \\ Séraphin. Un homme et son péché, Le Survenant and Aurore}

Abstract - Genre films are generally an excellent reflection of the dominant values of their time. Historical films, then, can constitute a very interesting field of investigation for one looking to understand the relationship of an era to the past. The films Séraphin. Un homme et son péché (2003), Le Survenant (2005), and Aurore (2005) mark a return of "classics" in Quebécois

$$
+4
$$

1. L'auteur tient à remercier les évaluateurs anonymes de Globe pour leurs judicieux commentaires, ainsi que les professeurs E.-Martin Meunier et Catherine Côté pour leur lecture atcencive ex leurs remarques constructives. 
cinema, and were analyzed in this perspective. What emerges from these three films is the astonishing persistence of the idea of the Québecois "Great Darkness". Above all, these films testify to a troubled relationship with religious heritage. The Québécois religious experience is distorted and the role of the Church and its clergy is quite harshly judged.

$+4+4+4+4+4+4+4+4+4+4+4$

La question du rapport au passé, à l'histoire et au temps, toujours centrale dans la définition d'une identité nationale, a beaucoup intéressé les spécialistes du cinéma québécois. Dans un essai de psychologie collective publié quelques mois après la sortie de Jésus de Montréal ${ }^{2}$, Heinz Weinmann pressentait l'avènement d'un nouveau rapport au passé, plus sensible aux défis du présent. Véritable "chef-d'œuvre" selon lui, le film de Denys Arcand permettait d'envisager une relation plus sereine avec la religion des ancêtres. En renouant avec "l'imitation de la Passion du Christ, mais hors de l'institution de l'Église ${ }^{3}$ ", les Québécois, espérait Weinmann, pourraient faire la paix avec leur passé et ainsi retrouver une certaine foi en eux-mêmes. Aussi, en assumant mieux le "message révolutionnaire d'Amour, de don de soi de Jésus", ils seraient davantage en mesure de s'ouvrir aux autres et de faire face au défi du pluralisme contemporain ${ }^{4}$. Dans une étude plus récente du cinéma québécois, Bill Marshall entrevoyait lui aussi un nouveau rapport au temps, à la fois post-traditionnel et postmoderne. Un film comme Le confessionnal ${ }^{5}$, de Robert Lepage, représentait selon Marshall une sortie définitive des grands récits théocratiques et modernistes qui avaient dominé le cinéma québécois des décennies précédentes. Ce film présentait l'écoulement du temps comme une succession de présents contingents et ouverts ${ }^{6}$.

Ces fins observateurs de l'évolution du cinéma québécois ne pouvaient prévoir le retour de "classiques" ${ }^{7}$ comme Séraphin. Un homme et

$$
+4
$$

2. Denys ARCAND, Jésus de Montrél Québec, 1989, 120 min.

3. Heinz WeInManN, Cinéma de l'smagznatre québécois. De La petıte Aurore à Jésus de Montréal, Montréal, L'Hexagone, 1990 , p. 25.

4. Ibid., p. 261.

5. Robert LEPAGE, Le confessionnal, Québec, 1994, 101 mın.

6. Bill MARSHaLl, Quebec Nattonal Cinema, Montréal/Kingston, McGıll-Queen's Universıty Press, 2001, p. 311. Sur le rapport du cinéma québécoıs au passé, vour aussı Christian POIRIER, "Le "renouveau" du cinéma québécois", Caté, n²3, Paris, Presses universiaires de France, 2005, p. 165-185.

7. Par "classiques", nous entendons, à la suite de Robert Melançon, des récits *anciens ressausis par le regard rétrospectif des modernes, des anciens relus et transformés par certe relecture qui redeviennent modernes» (Robert MELANÇON, Qu'est-ce qu'un classique québécots? Montréal, Presses de l'Université de Montréal/Fides, 2004, p. 15.). En plus d'être des récits anciens constamment réactualisés, les récits "classiques» se discınguent par leur grande popularité, peu importe le moyen de diffusion (livre, thêatre, radıo, télévisıon, cınéma). 
son péche $e^{8}$, de Charles Binamé, Le Survenant ${ }^{9}$, d'Érik Canuel, et Aurore $^{10}$, de Luc Dionne. Avec le recul, ces trois films forment un cycle qui s'apparente à celui qui avait été offert au public au tout début des années 1980, avec Les Plouffe ${ }^{11}$, Maria Chapdelaine ${ }^{12}$, tous deux réalisés par Gilles Carle, ainsi que Bonheur d'occasion ${ }^{13}$, de Claude Fournier. Comme ce premier grand cycle historique, les classiques récents offrent des terrains d'investigation tout à fait intéressants pour qui cherche à explorer le rapport qu'entretiennent les Québécois avec leur passé. Phénomène aussi spectaculaire qu'inattendu, ce retour des classiques a suscité beaucoup d'intérêt, tant chez la critique que chez le grand public. Si beaucoup de choses ont été écrites sur l'un ou l'autre de ces films, un regard d'ensemble sur ces trois productions sera particulièrement révélateur.

Dans l'essai qui suivra, je souhaite démontrer que les films analysés continuent d'alimenter la sombre perception d'une "Grande noirceur " passée ${ }^{14}$. Comme tous les films de genre, ces trois productions en disent plus long sur notre époque que sur ces ancêtres lointains, qui restent, pour bien des Québécois d'aujourd'hui, des ombres anonymes et sans relief, de purs étrangers avec qui le dialogue semble rompu. Conformes aux préjugés de notre époque, ces films historiques repoussent dans le passé toute la radicalité du Mal. Pour illustrer ce malaise, nous nous attarderons surtout à la place occupée par l'Église catholique et son clergé et au rapport général au phénomène religieux.

\section{LE GENRE HISTORIQUE}

Le succès de Séraphin. Un homme et son péché, du Survenant et d'Aurore doit être situé dans un contexte d'essor exceptionnel du cinéma québécois. Des films comme Les invasions barbares ${ }^{15}$ ou C.R.A.Z.Y. ${ }^{16}$ ont fait courir les foules et ont raflé de nombreux prix au Québec et à l'étranger. En 2005, les films québécois présentés en salle ont obtenu 18,2\% des parts de marché, un record. Même si le succès et les recettes ne sont pas toujours au rendez-vous,

$$
+4+
$$

8. Charles BINAME, Seraphin. Un homme et son péche, Québec, 2002, $128 \mathrm{~min}$.

9. Érık CANUEL, Le Survenant, Québec, 2005, 138 min.

10. Luc DiONne, Aurare, Québec, 2005, $115 \mathrm{~min}$.

11. Gilles CarLE, Les Plouffe, Québec, 1981, 227 min.

12. Gilles CarLe, Matia Chapdelanne, Québec, 1983, $105 \mathrm{~min}$.

13. Claude Fournier, Bonheur d'occaston, Quebec, 1984, 123 min.

14. Dans l'historiographie et la sociographie québécoises, la «Grande noirceur * est associée à la période de l'histoire québecoise qui va de l'Acre d'Union (1840) à la Révolution rranquille (1960).

15. Denys ARCAND, Les invastons barbares, Québec, 2003, $112 \mathrm{~min}$.

16. Jean-Marc VALLEE, C.RA.Z. Y., Québec, 2005, $129 \mathrm{~min}$. 
le cinéma québécois peut devenir une affaire rentable. Pour préserver l'engouement du public et battre ainsi les Américains sur leur propre terrain ${ }^{17}$, les producteurs tiennent désormais à présenter plusieurs genres de films: comédie, suspense, fantaisie, etc. L'objectif est d'offrir des produits diversifiés et d'ainsi répondre aux attentes des consommateurs ${ }^{18}$.

Comme c'est presque toujours le cas avec le cinéma de genre, les trois films ont été initiés par des producteurs ou des distributeurs, non par des scénaristes ou des réalisateurs. Lorraine Richard, productrice de Séraphin, l'admettait d'ailleurs candidement lors d'une entrevue accordée au Journal de Québec. Létincelle serait venue de Guy Gagnon d'Alliance Atlantis Vivafilm qui, lors d'un lunch, aurait lancé: «Ce serait l'fun de tourner un film qui serait quelque chose comme un classique ${ }^{19}$." Après deux tentatives ratées, Téléfilm Canada a finalement accepté de financer le projet et la réalisation du film a été confiée à Charles Binamé, reconnu pour sa trilogie urbaine ${ }^{20}$. Le projet est ambitieux, mais les coûts de réalisation, toutes proportions gardées, restent raisonnables puisqu'il s'agit d'un film historique : 6 à 7 millions de dollars ${ }^{21}$. Le Survenant et Aurore sont des productions similaires, car elles sont aussi entreprises par des producteurs. Les scénaristes et les réalisateurs ont en quelque sorte été choisis pour respecter le "cahier de charges" du producteur $^{22}$. Si Charles Binamé et Érik Canuel ${ }^{23}$ étaient des cinéastes respectés, le choix de Luc Dionne, pour qui Aurore était un premier film, a été vivement critiqué par le milieu du cinéma ${ }^{24}$.

Autre signe d'un cinéma de genre, ces trois films ont tous bénéficié d'un battage publicitaire très important ou, pour le dire comme la critique Odile Tremblay du Devoir, d'un «irritant bulldozer promotionnel${ }^{25}$ ". Plus d'un an avant la sortie en salles, le public a eu droit à une série

$$
++4
$$

17. Pierre BARRETTE, «Made in Quebec. Le cinéma québécois, encre imıtation et critique du modèle hollywoodien , 24 Images, $\mathrm{n}^{\circ} 128$, seprembre 2006 , p. 17-18.

18. Voir, la-dessus, Simon GALIERO, "Indusrrie er succes", Hor Champ, mars 2005, hrtp://www.horschamp.qc.cal article.php3?id_article=171 (5 décembre 2007).

19. Paul VilleneuVE, "Séraphin, Donalda et Alexis au grand ecran ", Journal de Québec, 10 novembre 2001, p. 66.

20. Charles BıNAMÉ, Eldorado, Québec, 1995, $104 \mathrm{~min}$; Le coutur au poing, Québec, 1998, $97 \mathrm{~min}$; La beauté de Pandore, Québec, 2000, $92 \mathrm{~min}$.

21. On est bien loin des 30 millıons accordés à la production de Nouvelle-France (Jean BEAUDIN, Nouvelle-France, Québec/France/Royaume-Uni, 2005, 245 min), un échec cuisant.

22. Denis COTE, "Le temps d'une paix", ICh, 21 avril 2005, p. 20.

23. Érik CANUEL, La lot du cochon, Québec, 2001, $98 \mathrm{~min}$; Nez rouge, Québec, 2003, $110 \mathrm{~min}$; Le dernter tunneh Québec, 2004, 109 min.

24. Vorr, à ce sujet, Germain LACASSE, "Les cinéastes québécois dans le contexte néolibéral, ou Grandeur et misère du

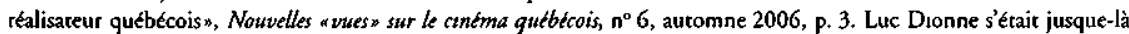
discingué en tant que scénariste de séries télévisées, avec Omertà (1996 à 1999) et Bunker - Le curque (2002).

25. Odıle TremblaY, "S'ouvrir à l'autre", Le Devoir, 23 avril 2005, p. E7. 
d'entrevues avec les producteurs et les réalisateurs. L'annonce des acteurs et des actrices qui allaient incarner les rôles mythiques de Séraphin, de Donalda, du Survenant, d'Angélina et de la marâtre martyrisant la petite Aurore a été savamment orchestrée de façon à rappeler aux gens la sortie prochaine du film. Au fil des reportages souvent racoleurs, le public en venait graduellement à confondre ces acteurs, ces actrices et les personnages qu'ils allaient incarner. Très connus du public, ceux-ci allaient d'ailleurs jouer un rôle clef dans la promotion du film en donnant de multiples entrevues. Dans le cas du personnage d'Aurore, des auditions ont été organisées par la productrice Denise Robert dans presque toutes les régions du Québec, ce qui a permis d'excellentes retombées médiatiques sur le plan local et régional. Près de 10000 fillettes auraient tenté leur chance, selon la productrice. Des émissions de variétés et des journaux à potins ont diffusé des images des lieux de tournage et des comédiens vêtus en costume d'époque. Les trois films ont aussi bénéficié de documentaires sur les coulisses du tournage diffusés à la télévision lors des périodes de grande écoute et dans lesquels on pouvait voir les réalisateurs et les acteurs raconter des anecdotes amusantes sur le tournage ou réfléchir au sens du film. Notons que les spécialistes des œuvres traitées ne semblent pas avoir été sollicités, sauf dans le cas d'Aurore. Les documentaires des coulisses du Survenant et d'Aurore effectuaient un retour sur les lieux du récit pour discuter avec des "gens ordinaires". Les critiques spécialisés ont pu, comme c'est la coutume, visionner le film avant la sortie en salles, mais ont été astreints à respecter un embargo plutôt rigide, de façon à minimiser l'impact d'une critique négative.

Cette campagne de promotion a donné des résultats plus que convaincants en matière d'assistance et de recettes. Véritable succès populaire, Séraphin. Un homme et son péché a même battu le record d'assistance établi par le film Les Boys en 1999. Une assistance de 1,3 million de spectateurs et des recettes de plus de 8 millions de dollars en font l'un des plus grands succès du cinéma québécois. À titre de comparaison, l'histoire de Séraphin Poudrier a attiré 500000 spectateurs de plus que C.R.A.Z.Y., 400000 de plus que Les invasions barbares, 150000 de plus que La grande séduction. Sans ce succès retentissant, Le Survenant et Aurore n'auraient sans doute jamais vu le jour. Ces deux films sont sortis à quelques mois d'intervalle, exactement trois ans après Séraphin. Un homme et son péché. Des deux, c'est Aurore qui a été le plus populaire, attirant 706000 spectateurs en 2005, soit 300000 spectateurs de plus que Le Survenant. On est loin du succès phénoménal de Séraphin; cela dit, Aurore a été plus populaire que Horloge biologique (160000 de plus), et Le Survenant a mieux réussi que des 
films "grand public» comme Les Boys 4 ou Idole instantanke. En somme, le pari des producteurs a été gagné. Le public a répondu à l'appel et les recettes furent appréciables ${ }^{26}$.

Par leur structure de production, l'ampleur de leurs campagnes promotionnelles et l'importance accordée aux acteurs, Séraphin, Le Survenant et Aurore ont tous les ingrédients d'un cinéma de genre. Comme le montre de façon convaincante Pierre Véronneau, ce type de cinéma a une longue histoire au Québec. Sa vitalité actuelle semble démontrer que le cinéma québécois a atteint un niveau de dynamisme et de maturité sans précédent ${ }^{27}$. Le genre à l'honneur ici est évidemment le genre historique, censé divertir autant qu'une comédie, un thriller ou un film pour enfants. Le danger du "cinéma de genre" est souvent d'offrir un "produit" bien fait, exemplaire sur le plan technique, mais sans originalité ni substance. Quant au genre historique, la tentation est grande d'offrir une série d'images d'Épinal dignes des "minutes du patrimoine" ou de cartes postales du bonvieux-temps ${ }^{28}$ et de présenter un récit attendu, conforme aux préjugés du temps. Ces faiblesses, facilement perceptibles dans chacun des trois films qui nous intéressent, que n'a d'ailleurs pas manqué de relever la critique, peuvent toutefois s'avérer un atout pour étudier le rapport au passé. Contrairement au cinéma d'auteur qui souhaite le plus souvent faire voir le monde autrement, convaincre de la justesse d'une cause ou subvertir les conventions du septième art, le cinéma de genre cherche l'adhésion du spectateur en lui présentant des personnages dans lesquels il pourra se reconnaître et un récit dont il pourra facilement s'approprier la trame. Si le cinéma d'auteur vise la mise à distance du spectateur par rapport à lui-même et à ce qui l'entoure, le cinéma de genre vise, au contraire, l'identification du spectateur avec ce qu'il voit à l'écran ${ }^{29}$. Pour gagner le spectateur au film, le producteur cherche souvent à s'approcher le plus possible de sa perception du monde. Par conséquent, le cinéma de genre constitue un excellent reflet des valeurs

$$
+\div
$$

26. Selon l'Observatoire de la culture et des communications du Québec, Aurore et Śraphon. Un homme et son péché auratent recueilli des recettes de 4,4 et de 2,9 millions de dollars. Voir http:/www.stat.gouv.qc.ca/ observatoire/publicat_obs/pdf/StatbrefNo18.pdf ( 5 décembre 2007).

27. Pierre VéronNEAU, $k$ Genres and Varıatıons. The Audiences of Quebec Cineman, André Loiselle et Tom MCSORLEY [dir.], Self Portrats. The Cinemas of Canada Since Telefilm, Ottawa, The Canadian Film Instirure/Institut canadien du film, 2006, p. 93-127.

28. Après avoir visionné Le Survenant, l'actrice Béatrice Picard, qui avaıt personnıfié Angélina dans la célèbre sérne rélévisée, déclare: "j'ai eu l'impression que tout au long de la soirée, on tournat pour moi les pages d'un beau livre d'historre remplie d'ımages d'Epınal sougnées à farre rêvern (cutée dans La Presse, 16 avril 2005, p. D2).

29. Pierre VéronNeAU, op. cat., p. 121. 
dominantes d'une époque, tout comme les séries populaires de la télévision ${ }^{30}$. En plus de présenter au public des personnages attachants et un récit captivant, un film qui fonctionne bien en salles se conforme aux perceptions dominantes ou, en d'autres termes, correspond aux horizons d'attente du public.

On peut donc penser que l'immense succès de Séraphin. Un homme et son péché et d'Aurore tient à une représentation du passé canadienfrançais tout à fait conforme à celle qui domine notre époque. Ces deux films confortent le spectateur dans ses valeurs. Après les avoir visionnés, il ne peut en effer que se réjouir d'être sorti de cette époque d'intolérance, de rigorisme, de fermeture d'esprit, de sacrifice et de misère. Il ne peut que maudire les ténèbres de la "Grande noirceur». Avant la sortie du film, Charles Binamé et Luc Dionne avaient d'ailleurs beaucoup insisté sur la contemporanéité et l'universalité du propos de leurs films. Deux ans avant la sortie en salles de Séraphin, Binamé promettait "un Séraphin Poudrier loin du folklore ${ }^{31}$ ", plus proche des préoccupations d'aujourd'hui. Séraphin, expliquait Binamé, est "à sa façon une multinationale qui tient tout le monde en son pouvoir ${ }^{32}$ ". Si le réalisateur énonçait son intention de montrer le travail éreintant des colons des pays d'en haut, il souhaitait du même souffle dénoncer "le grand projet du curé Labelle", "une honte monumentale ${ }^{33}$ " selon lui. De son côté, Luc Dionne insistait sur le fait qu'Aurore est un drame qui traite des enfants battus et maltraités. "Ce n'est pas un film d'époque, précise l'actrice Hélène Bourgeois-Leclerc qui personnifie la marâtre, c'est un film d'actualité dans un décor d'époque ${ }^{34}$." Le film sort d'ailleurs à un moment où la Direction de la protection de la jeunesse (DPJ) est l'objet de nombreuses critiques ${ }^{35}$. Cette insistance visait à rassurer un public qu'apparemment seul le présent intéresse. Le passé, dans ce qu'il a de différent et de dépaysant, est mis sous le tapis lors de la promotion de ces deux films. Même dans le cas du Survenant, film pourtant plus fidèle à l'œuvre originale, certains insistent sur l'actualité du propos. D'aucuns y voient un film sur "la différence et sur l'acceptation de l'autre (et de soi) ${ }^{36}$ ", alors que d'autres comparent Le Survenant à un

$$
\div+
$$

30. Voir Jean-Pierre DeSAULNIERS, De la famille Plouffe à La perire vie. Les Quebtcois et leurs teleromans, Montréal/Québec, Musée des civilisations/Fides, 1996.

31. Charles BINAmÉ, ciré par Gilles CARIGNAN, "Le tetour de Séraphin *, Le Soleth 19 février 2000, p. C3.

32. Charles BinamÉ, ciré par Chantal GuY, "Un homme er son péché, l'intégralen, La Presse, 15 octobre 2001, p. Cl.

33. Charles BINAME, ciré par Gilles CARIGNAN, «Le pari de Séraphinm, Le Soleil, 23 novembre 2002, p. G4.

34. Hélène Bourgeois-LeClerC, cité par Paul VilLeNeuve, a Le drame d'Aurore ", Le Journal de Québec, 29 juin 2005 , p. 69.

35. Ces critiques proviennent notamment du journaliste Paul ARCAND, réalisateur d'un documentaire à sensation, Les voleurs d'enfance (Québec, 2005, $88 \mathrm{~min}$ ), diffusé dans les salles de cinéma la même année.

36. Gilles CarignaN, a Le Survenant. Au-delà de la recerten, Le Soleih 23 avril 2005, p. G3. 
membre de la génération $\mathrm{X}^{37}$, "plus grouillante et désireuse de voir du Pays $^{38}$ ". Érik Canuel est cependant le seul à n'avoir pas joué cette carte lors de la promotion de son film. Ce réalisateur, dont le père acteur Yvan Canuel "affichait un véritable engouement pour nos racines ${ }^{39}$ ", affirme avoir suivi "l'inspiration poétique du roman ${ }^{40}$ ".

En comparaison avec Séraphin et Aurore, la critique sérieuse a été beaucoup plus clémente à l'endroit du Survenant, un film qui respecte davantage le classique littéraire dont il s'inspire et l'époque concernée. Or, c'est le film qui a eu le moins de succès en salles. Le Survenant, plus empathique et un brin nostalgique, correspondait vraisemblablement moins aux attentes du public d'aujourd'hui que Séraphin et Aurore, qui ont su toucher les bonnes cordes.

Ces trois films ont beaucoup en commun : le point de vue dominant est celui d'une victime de sexe féminin (Donalda, Aurore et Angélina). Ces femmes sont, dans les trois cas, les innocentes victimes d'une communauté repliée sur elle-même, ayant la paroisse ou le village pour seul horizon et qui se méfie de tout ce qui vient d'ailleurs. Les habitants de ces communautés paraissent sans relief: le plus souvent, ils sont dépeints comme des êtres dociles qui ne contestent pas l'autorité, jacassent à propos de choses sans importance et médisent des uns et des autres. Des êtres "pittoresques" mais un peu niais, sans aucune envergure ni sagesse, qui tolèrent l'intolérable et détournent le regard lorsqu'un drame se produit sous leurs yeux. Ils obéissent ainsi, sans sourciller, tant par couardise que par lâcheté, à une inacceptable loi du silence. Dans les communautés traditionnelles, laisse-t-on chaque fois entendre, cette omerta était la norme; il fallait s'y plier sous peine d'être exclu. Les films de Charles Binamé et d'Érik Canuel cherchent cependant à montrer, à travers les personnages d'Alexis et du Survenant, que cette pesante tradition est battue en brèche par des êtres libres qui défient les conventions. Le personnage d'Alexis Labranche, joué par Roy Dupuis dans Séraphin, ressemble d'ailleurs plus au François Paradis de Maria Chapdelaine qu'au Alexis de Claude-Henri Grignon, installé sur une terre et père de huit enfants. Ses rêves de liberté, son indépendance d'esprit et ses ambitions pour un pays qui regorge de richesses font de lui, et non plus du curé Labelle,

$$
+4
$$

37. Ou génération "post-boomers", née encre 1961 ec 1981.

38. Émilie GAUTHIER-SIROIS, "Un délıce d'antan", Le Courrier, 27 avril 2005, p. B2.

39. Érık CANUEL, cité par Marc-André LusSIER, "Le Survenant ressuscitée, La Presse, 16 avril 2005, p. C2.

40. Érik Canuel, cıté par Paul Vilueneuve, "Le Survenant. Un grand défi pour Canuel", Journal de Québec, 16 avril 2005, p. 22. 
comme dans l'œuvre originale, le véritable héros du film. Si le Survenant et Alexis incarnent la modernité à venir, rien de tel dans Aurore. Sur ce plan comme sur bien d'autres, le film de Luc Dionne apparaît comme le plus sombre. Le monde qu'il décrit est clos, fermé et noir jusqu'à l'extrême. L'air y est irrespirable. Aucun espoir en vue.

\section{UN RAPPORT TROUBLE À L'HÉRITAGE CATHOLIQUE}

Les films Séraphin et Aurore partagent surtout une vision extrêmement sombre du rôle joué par l'Église catholique et son clergé dans le Canada français de la Grande noirceur. À bien des égards, ces films reprennent à leur compte le lourd contentieux des générations qui ont soutenu la révolution culturelle des années 1960. L'Église est représentée comme une institution oppressante, surtout pour les femmes qui sont confinées à leur rôle de mère et toujours obligées de se sacrifier. Cette représentation négative, voire caricaturale, n'est probablement pas étrangère au succès des deux films. Chose certaine, elle tranche avec la représentation du religieux que l'on retrouve dans le premier cycle du genre historique offert au début des années 1980.

Dans Les Plouffe, Maria Chapdelaine et Bonheur d'occasion, l'Église catholique et le phénomène religieux sont présentés avec distance et respect. Les rites pieux pratiqués par les familles Plouffe et Chapdelaine ne sont pas ridiculisés. L'éthique religieuse traditionnelle des personnages, rarement évoquée, renvoie à l'ancienne religion des Canadiens français, celle qui prévalait avant Vatican II. Cette culture religieuse insistait sur la condition pécheresse de l'homme, affirmait l'existence d'un ordre naturel immuable et accordait au clergé une place prépondérante dans tout ce qui concernait la sphère spirituelle ${ }^{41}$. Dans Bonheur d'occasion, cette éthique traditionnelle est vécue sur le mode de la résignation face aux malheurs. Elle permet de comprendre l'attitude d'Yvonne, la sœur cadette de Florentine, qui accepte sereinement la mort de son petit frère Daniel, ou les lamentations de la mère Lacasse, qui se montre fataliste face au mauvais sort qui s'acharne presque toujours sur le "pauvre monde». Cette résignation est aussi celle de Samuel Chapdelaine lorsqu'il apprend la mort de François Paradis: «On est tous des enfants dans la main de Dieu." Dans Les Plouffe et Maria Chapdelaine, le

$$
4+4
$$

41. En plus d'être contestée par les parcisans d'une moderntté lauque, sinon athée, cette échique catholique craditionnelle a aussi été remise en question à l'intérıeur même de l'Église catholique par les tenants d'une nouvelle éthique dite "personnalistes. Voir E.-Marcin MEUNIER, Le pan personnaluste. Modernite et catholicasme au XX" siecle, Montreal, Fides, 2007. 
clergé occupe une place plus importante que dans Bonheur d'occasion. Personnifiés par Gérard Poirier et par Claude Rich, le curé Folbèche et le père Cordelier sont des personnages plutôt rigides qui défendent avec conviction un certain ordre moral et leur Église. S'ils valorisent l'obéissance à la parole des évêques et vivent à l'heure du Vatican, ils savent cependant s'adapter aux circonstances et s'ouvrir aux nouvelles réalités du monde. En effet, le curé Folbèche ne craint pas de s'initier au baseball, et les ursulines, parmi lesquelles certaines apprennent à faire du patin à roulettes, acceptent d'enseigner le français à la femme d'un pasteur protestant. Il en est ainsi parce que ces clercs sont présentés comme des gens proches de leurs concitoyens. On les voit finalement assez peu en chaire en train de prêcher. Ils prodiguent davantage des conseils qu'ils ne donnent des ordres. Leur langue est pure, formelle, mais reste accessible à tous. Lorsqu'on les compare aux trois classiques remis au goût du jour, ces films historiques des années 1980 ne cherchent pas à régler de comptes avec l'Église: les réalisateurs Gilles Carle et Claude Fournier n'ont pas souhaité noircir le tableau, mais bien rendre compte d'un certain rapport au religieux et à la transcendance. Le religieux est présenté comme l'une des dimensions de la culture canadiennefrançaise.

Le rapport au passé religieux que traduit le film d'Érik Canuel est, de loin, le plus sobre. Fidèle au roman de Guèvremont, il présente le Survenant tel un envoyé de la Providence qui sème l'espoir dans une communauté endeuillée $e^{42}$. Comme dans la télésérie étudiée par Jean-Pierre Desaulniers, il est une sorte de "messager divin, un ange, une incarnation troublante du bien et du beau ${ }^{43}$ ". Au contact du Survenant, Phonsine devient plus sûre d'elle et tombe enceinte, le père Didace fait le deuil de son fils Éphrem mort noyé et rencontre l'amour, Angélina cesse de se percevoir comme une boiteuse ou comme une fille bonne à marier à cause de sa dot. Le personnage du Survenant d'Érik Canuel semble être une figure christique qui, dans la première et la dernière image du film, suit une croix. Comme Jésus, le personnage vit parmi les hommes, partage leurs souffrances et certaines de leurs faiblesses. Lorsqu'il rentre ivre de l'une de ses tournées à Sorel, il échappe de sa poche un crucifix que s'empresse de ramasser la pieuse Phonsine, ce qui rappelle l'aura religieuse du personnage. S'il respecte le message de fond du roman de Guèvremont, Canuel omet toutefois certains

$$
++4
$$

42. À la fin du film, on peut voir cette citation tırée de la Bible: «Celui qui marche dans les rénèbres ne sait pas où il va. Croyez en la lumière afin que vous soyez des enfants de lumière" (Érik CANUEL, Le Survenant, Québec, 2005, $138 \mathrm{~min}$ ). 43. Jean-Pietre DESAULNIERS, op. cit., p. 49. 
éléments liés au rapport à l'héritage religieux. Dans le roman, Angélina est présentée comme une personne très pieuse; elle aurait même songé à devenir sacristine, ce qui permet de mieux comprendre ses hésitations à prendre mari et son austérité. Aucune scène du film ne montre la communauté du Chenal du Moine à l'église, ni même l'église tout court. Le personnage du curé Lebrun, à qui le père Didace demande la permission de se remarier avec la Cayenne, n'existe pas. Jamais confronté à l'Église réelle, avec ses rituels, ses prescriptions et son clergé qui veille au grain, Le Survenant apparaît comme le seul véritable rédempteur ${ }^{44}$. L'église est la grande absente de ce film, ce qui tranche avec Séraphin et Aurore, beaucoup plus populaires en salles.

La religion et la morale sont au cour du roman Un homme et son péché. Le véritable drame du Séraphin Poudrier mis en récit par ClaudeHenri Grignon est d'être l'esclave de l'un des sept grands péchés capitaux: l'avarice. Sa cupidité est plus forte que la faim, le froid et l'amour. Le héros de Grignon est également tenté par le péché de luxure, mais dans une moindre mesure. On comprend, en lisant le roman, que ces deux péchés capitaux sont à l'origine de son mariage avec Donalda: "Ses hautes qualités de paysan retors le poussaient à rechercher, dans la femme, la bête de travail beaucoup plus que la bête de plaisir. Comment aurait-il pu hésiter puisqu'il posséderait les deux ${ }^{45}$ ? "Toute l'intrigue de cette histoire tourne autour de ce péché et non pas, comme dans le film de Binamé, d'une histoire d'amour impossible. Grignon raconte l'histoire d'un homme incapable de résister à son péché et cette incapacité lui donne une dimension plus humaine. Derrière sa toute-puissance apparente, Séraphin est, au fond, un homme fragile qui succombe à un péché ignoble. Cette dimension humaine, parfois même affable, du personnage est cependant absente du film de Binamé. La première scène du film, durant laquelle le jeune Séraphin surprend sa mère en train de se prostituer pour quelques sous, suggère que le mécanisme de cette perversion trouverait son origine dans l'enfance. Binamé a beau s'enorgueillir d'avoir conservé le titre original par souci de ne pas "gomme[r] notre mémoire ${ }^{46}$ ", il privilégie l'explication psychologisante et passe complètement à côté de la notion de péché, centrale à l'histoire originale. Dans la religion traditionnelle des Canadiens français, telle que décrite par

$$
+++
$$

44. François MaCcabé IQbal, "Survenant" le rédempreur*, Cécile Cloutier-WojCIECHOWSKa et Rejean RoBIDOuX [dir.], Solinude rompue Hommage à David M. Hayne, Otcawa, Presses de l'Universıté d'Ottawa, 1986, p. 248256.

45. Claude-Henti Grignon, Un bomme et son peché, Montréal, Centre éducatif et culcurel, 1965 [1933], p. 12.

46. Charles BINAME, cité par Gilles CaRIGNAN, «Le pari de Séraphin ", op. cit, p. G4. 
Benoit Lacroix, la crainte du péché était omniprésente ${ }^{47}$. Outre les dix commandements de Dieu, qu'il fallait respecter à la lettre, le fidèle devait suivre les sept commandements de l'Église, se prémunir contre les sept péchés capitaux et obéir à toute une série de prescriptions ponctuelles. Les péchés commis, volontairement ou par inadvertance, devaient être confessés et profondément regrettés par tous les croyants. D'une certaine façon, le roman de Grignon témoigne de cet aspect fondamental de la religion traditionnelle des Canadiens français. Or, Binamé a consciemment choisi d'omettre du film cet aspect de la culture religieuse d'autrefois parce qu'il fallait «faire en sorte que les jeunes puissent s'y reconnaître. Il n'y aura pas seulement des bondieuseries et des signes de $\mathrm{croix}^{48}$ ". Binamé confond ici «bondieuseries» et foi, rituels et croyances. En ne nous permettant pas de comprendre le sentiment de culpabilité lié au péché, Binamé rend le caractère du personnage de Séraphin totalement opaque et unidimensionnel.

Cette incapacité à rendre compte de l'idée de péché ne nous permet pas non plus de saisir le drame intérieur que vit Donalda. Dans le roman, la jeune épouse de Séraphin est une femme extrêmement pieuse, membre de l'Union des prières de la paroisse. Pour elle comme pour bien des femmes de son temps, le mariage est un serment sacré fait devant Dieu. Une fois celui-ci scellé, on ne pouvait revenir en arrière. Il fallait accepter son sort et travailler inlassablement à l'harmonie du foyer, fut-ce en s'oubliant et en se sacrifiant. Dans les sociétés traditionnelles, le motif principal du mariage n'était pas la compatibilité entre l'homme et la femme ni même l'amour, mais la reproduction à l'intérieur d'une institution stable sur laquelle reposait l'ordre social. La piété de Donalda, sa résignation devant les malheurs qui s'abattent sur elle n'ont de sens que si on les situe dans un contexte où dominait l'éthique catholique traditionnelle évoquée plus haut. Étrangère à notre temps, choquante même à bien des égards, la foi de Donalda correspond à une façon traditionnelle de vivre sa foi. Dans cette optique, le sacrifice pouvait avoir un sens parce qu'il permettait de se purifier de ses péchés, de "gagner son ciel". Les dirigeants de la Société de développement des entreprises culturelles (SODEC) craignaient que le personnage de Donalda ne provoque une réaction hostile du public féminin, ce qui aurait nui au succès du film ${ }^{49}$. Charles Binamé et l'actrice Karine Vanasse ont donc tenté de

$$
++
$$

47. Benoit LACROLX, "Le temps du péché", La foi de ma mère La religion de mon père, Montréal, Bellarmin, 2002, p. $113-128$.

48. Paul ViLleNEUVE, "Séraphın, Donalda et Alexis au grand écran ", op. cat., p. 66.

49. Paul VILLENEUVE, " "Un homme ex son peché. Une histoire qu'on ame” - Charles Binamé", Le Journal de Montréal, 17 novembre 2002, p. 37. 
contrer cette perception en présentant Donalda comme une femme volontaire, une héroïne même, qui aurait décidé de marier Séraphin Poudrier en toute connaissance de cause. En épousant Séraphin, elle efface les dettes d'un père qui, désespéré par ses soucis financiers, tente de se suicider; en acceptant de mourir, elle libère Alexis, en train de gâcher sa vie en l'attendant ${ }^{50}$. Contrairement à ce que craignaient les dirigeants de la SODEC, le personnage de Donalda ne semble pas avoir choqué le public; il a, au contraire, confirmé ses préjugés les plus tenaces sur le rôle néfaste de l'Église catholique, surtout auprès des femmes. Le comportement du personnage féminin, explique le critique Jean-Philippe Gravel, "rappelle un peu trop la soumission sous l'esclavage que cautionnait auprès des paroissiens notre Église catholique, pour qui un destin de martyr vous assurait un aller simple en première classe vers le Bon Dieu ${ }^{51}$ ». Pour la critique Marie-Josée Montminy, le drame de Donalda conforte même les féministes dans leur combat. Dans le Québec de la fin du XIX siècle, "la soumission à des forces sociales plus puissantes que la liberté individuelle» et "l'abandon de soi par sacrifice pour autrui" étaient la norme, la "mentalité d'une époque ${ }^{52}$ ". Alors que Binamé et Vanasse voulaient faire de Donalda une femme libre, une héroöne qui se sacrifie peut-être, mais par amour - pour son père et pour Alexis -, la réception montre qu'elle est plutôt perçue comme l'une des nombreuses victimes de l'Église. Son sacrifice est considéré comme le pur résultat d'une contrainte extérieure, non comme l'adhésion à une éthique différente de celle de notre époque. En présentant Donalda comme une femme spontanée, indépendante d'esprit, voire libérée (ses seuls rêves sont érotiques !), comme le fait Binamé, on transpose les attributs d'une jeune femme actuelle dans le contexte de la fin du XIX ${ }^{e}$ siècle. En ne nous permettant pas de saisir les raisons profondes de sa soumission, les ressorts intérieurs de sa foi, sa résignation apparaît comme une sorte de masochisme incompréhensible qui ne fait qu'attiser la colère des contemporains à l'égard des «forces sociales» de cette époque ${ }^{53}$.

Cela dit, faire de Donalda Laloge une victime de l'Église et de sa morale contraignante relève du pur préjugé d'époque puisque Binamé ne suggère pas cette interprétation dans son film. Le curé du village ne fait

$$
4+\div
$$

50. Isabelle MASSE, «Un classique revisité \#, La Presse, 23 novembre 2002, p. C6.

51. Jean-Philippe GraVEL, "Saga*, Ich, 4 decembre 2002, p. 13.

52. Marıe-Josée MONTMINY, «Vive le féminısme! n, Le Nouvelliste, 30 novembre 2002, p. A7.

53. Nous nous inspirons ici de l'analyse de Lysiane GAGNON, « Donalda. Erreur sur la personne”, La Presse, 28 décembre 2002, p. A13. 
pression sur elle d'aucune façon pour qu'elle épouse l'ignoble avare; il la laisse même libre de décider, en son "âme et conscience». Dans le roman régionaliste de Claude-Henri Grignon, on ne se surprend guère de voir le curé Raudin dépeint comme un saint homme, proche de ses paroissiens, généreux et prêchant par l'exemple. Cette bonté, cela va sans dire, lui vient de sa foi profonde, intense. Dans le film de Binamé, le personnage du curé est empathique et humain, ce qui est plutôt fidèle au roman, mais pour le moins étonnant par rapport à la mémoire de la Grande noirceur. Pour rendre la bonhomie de Raudin crédible et acceptable pour notre époque, Binamé en fait cependant un curé qui aurait roujours eu du mal à respecter son vœu de chasteté: vivant une liaison avec une dame du village, il attrape une maladie vénérienne et, à la toute fin, décide de défroquer pour vivre son amour au grand jour! En procédant ainsi, Binamé choisit le préjugé d'époque. Plutôt que de tenter de rendre compte de l'engagement profond d'un homme généreux qui vit intensément sa foi et ainsi nous dépayser ou nous renvoyer autre chose qu'un miroir de nous-mêmes, il nous présente un bon bougre, pas méchant pour deux sous. Plus proche de l'ami qui console que du curé qui rassure, le personnage finit par défroquer parce qu'il a su trouver le "courage", comme si quelqu'un l'avait obligé à devenir prêtre.

En présentant Séraphin comme l'avare qui tient le rôle du méchant, Donalda comme une jeune femme qui se sacrifie sans raison et le curé comme un coureur de jupons, Binamé et son scénariste évacuent complètement le fait religieux au cœur de cette histoire de péché et de sacrifice. Du religieux, on ne retient que les rituels pratiqués par habitude et par conformisme social, et on évacue le combat intérieur de Séraphin ainsi que la foi de Donalda et du curé. Tout se passe comme si le fait religieux était totalement extérieur aux personnages.

Dans le cas de Séraphin et du Survenant, il est possible de se référer aux romans originaux pour voir si les cinéastes revisitent l'œuvre ou travestissent complètement l'intention des auteurs ${ }^{54}$, ce que Charles Binamé me semble faire. Dans le cas d'Aurore, Luc Dionne disposait d'une plus grande liberté puisque cette histoire repose sur un fait divers, une pièce de théâtre très populaire et le film de Jean-Yves Bigras de $1952^{55}$ qui, en dépit de ses évidentes faiblesses, a connu un important succès depuis sa sortie ${ }^{56}$. Dans

$$
+\div
$$

54. Nous aurions évidemment pu nous référer aux téléséries qui ont sûrement fait évoluer les récics de manière à les rendre plus conformes à l'esprit du remps, mais nous avons préféré nous en tenir aux romans originaux. 55. Jean-Yves Bigras, La petite Aurore l'enfant martyre, Québec, 1952, 105 min.

56. En 2005, la compagnie Equinoxe jugeait le film suffisamment populaire pour en farre un DVD 
le commentaire qu'il émet à propos de son film, disponible sur DVD, le scénariste et réalisateur Luc Dionne dit s'être inspiré d'un roman d'André Mathieu ${ }^{57}$ et avoir effectué des recherches afin de se rapprocher le plus possible de la vérité historique. Pour rendre la dramatisation plus convaincante, il admet cependant avoir pris certaines libertés, de sorte que l'histoire qui nous est présentée se veut clairement une interprétation très personnelle du drame d'Aurore Gagnon. Lorsqu'on y regarde de plus près, la thèse de Luc Dionne est assez simple, sinon caricaturale. Le véritable coupable du meurtre d'Aurore n'est pas la marâtre Marie-Anne Houde, comme le démontre clairement le film de Jean-Yves Bigras, ni même le village qui attend trop longtemps avant d'intervenir, mais bien l'infâme curé Leduc et l'institution qu'il représente, l'Église catholique.

Alors qu'entre 1914 et 1920, le village où vécut Aurore a vu défiler trois curés, Luc Dionne a préféré n'en présenter qu'un. Incarné par l'excellent Yves Jacques, le curé Leduc est la caricature même du curé autoritaire, pédant, moralisateur et "incarnant à lui seul tout ce que le clergé pouvait représenter de plus éloigné de la réalité terrestre ${ }^{58}$ ", selon la journaliste Valérie Lessard. L'homme est toujours dans ses livres, cite de mémoire des locutions latines et des écrivains français, et vit en dehors du monde réel de ses paroissiens qui triment dur comme Télesphore, le père d'Aurore. Il sermonne davantage qu'il ne guide et pérore davantage qu'il ne conseille, dans une langue ampoulée, affectée et évidemment très loin de celle de ses paroissiens. Il joue de cette distance avec le peuple non pour préserver le mystère de sa fonction, mais pour imposer ses diktats et son pouvoir. Les mots, la culture et le sacerdoce sont pour lui des instruments de contrôle social avant tout. Le curé Leduc est un individu sans humanité dont les croyances sont purement intellectualisées, livresques. Le personnage, «inauthentique» par excellence, rappelle l'analyse que proposait Jean Larose à propos du Déclin de l'empire américain ${ }^{59}$. Dans l'imaginaire québécois, les hommes cultivés sont souvent mesquins et efféminés, c'est-à-dire coupés de ce qui devrait être "vrai" chez un homme: le désir sexuel, la force et la vulgarité. À la fois curé et intellectuel, le personnage est l'inauthentique par excellence, il représente un "beau cas " pour qui cherche à sonder l'inconscient collectif des Québécois.

$$
+\div
$$

57. André MATHIEU, Aurore, La vraie histoure, Sant-Eustache, Edition du Cygne, 1990.

58. Valérie LESSARD, "Aurore". Un direct au coeurn, Le Drott, 9 juillet 2005, p. A6.

59. Jean LAROSE, "Savoir et sexe dans Le déclen de l'empire américain", La perite noirceur, Moncréal, Boréal, 1987, p. $12-14$. 
Luc Dionne montre que le curé Leduc se retrouve dans le village d'Aurore sans l'avoir choisi. Docteur en théologie et homme de pouvoir, il rêvait plutôt d'œuvrer au Vatican, mais ses supérieurs ne l'entendaient pas ainsi et ont préféré lui confier une petite paroisse des Bois-Francs. "Vous détestez ceux qui réfléchissent", lance le curé Leduc à son supérieur lorsqu'il apprend la décision. L'Église, en tant qu'institution, est d'une certaine façon à l'origine du malheur de la petite Aurore parce qu'elle instille l'amertume chez un homme qui avait choisi un autre destin. Une fois sur place, le curé Leduc pose un certain nombre de gestes qui font de lui le véritable coupable du film. Il manque d'empathie à l'égard de Télesphore, qui vit le deuil de sa première femme. Au lieu de lui venir en aide, le prêtre lui reproche de ne plus pratiquer ses rituels religieux et surtout, il condamne sans appel sa situation de concubinage avec Marie-Anne Houde, une cousine devenue veuve. Pour sauver les apparences, il l'oblige à rapatrier ses enfants à la maison, alors qu'ils étaient jusque-là dispersés chez la famille pendant que leur mère était à l'hôpital. Aurore est ainsi arrachée du milieu familier et protecteur qu'est celui de ses grands-parents maternels. Avec le retour des enfants du premier lit, les problèmes commencent: les plus jeunes enfants meurent mystérieusement. Au lieu de s'inquiéter, le curé Leduc accepte le récit cousu de fils blancs de la marâtre et condamne les mauvaises langues qui font courir toutes sortes de rumeurs. Lorsque d'autres rumeurs font état de sévices administrés à la petite Aurore, le curé Leduc accepte à nouveau l'explication tordue de la marâtre. Cette dernière rapporte qu'Aurore serait l'enfant du péché et qu'elle aurait été conçue dans la luxure et l'alcool. Cette origine malsaine expliquerait le caractère difficile de la fillette qu'il faut à tout prix "corriger». De telles "corrections", montre Luc Dionne, étaient bel et bien prescrites par le petit catéchisme, donc encouragées par l'Église et ses représentants. Hospitalisée pendant quelques semaines, la petite Aurore suscite la pitié d'une jeune religieuse. De retour dans la paroisse, Aurore est accusée par le curé Leduc d'avoir répandu des "mensonges» sur son état. À partir de ce moment, il dit mieux comprendre pourquoi il faut "corriger" Aurore, ce qui entraîne sa perte. Alerté par le juge de paix, porte-parole politique de la société civile, le curé Leduc refuse de prêter son concours. Seul l'appel d'un représentant de la Couronne à Québec le fera plier. Après le drame, le curé Leduc ne montre aucune forme de repentir. En chaire, il prêche plutôt l'oubli, ce que refusent ses paroissiens.

Soucieux de toujours sauver les apparences, complice de la marâtre qui souhaite "corriger" une enfant du péché et complètement indifférent au drame d'Aurore, alors qu'il aurait pu intervenir très tôt, le curé 
Leduc est le véritable coupable de cette sordide histoire. Même si la justice officielle va la condamner très sévèrement, la marâtre n'est pas celle qui se retrouve sur le banc d'accusé dans le film de Luc Dionne. Ce dernier suggère même qu'au contraire, la marâtre a elle aussi été une victime de l'Église. Dionne fait dire à l'un des enfants de Marie-Anne Houde que sa terrible cruauté était liée à des dépressions qu'elle vivait lors de ses grossesses. Or, qui obligeait les femmes à vivre ces grossesses à répétition, sinon l'Église? Qui condamnait sévèrement celles qui empêchaient la famille, sinon le clergé? Cette représentation de la marâtre comme victime sera reprise par certaines critiques:

L'Église porte encore son poids d'erreurs et de la souffrance des gens ordinaires à cette époque. On n'a qu'à songer au fardeau que représentaient les grossesses à la chaîne sous peine de péché mortel. La triste folie et la cruauté de Marie-Anne y prennent leur source. Dans la réalité, cette femme, mariée à 17 ans, a dû enfanter tous les ans, ce qui ne prépare pas à l'acceptation généreuse des enfants d'une autre ${ }^{60}$.

Alors que Luc Dionne se dit fidèle à la vérité historique, il admet qu'il triche lorsque c'est le juge de paix plutôt que le curé qui découvre le corps meurtri de la petite Aurore. Le verdict de culpabilité est prononcé à la toute fin du film par le juge de paix incarné par l'excellent Rémy Girard, lors d'un échange révélateur. Ivre de colère, la "colère antithéologique" des Québécois post-Révolution tranquille ${ }^{61}$, le juge de paix assène au curé ses quatre vérités. Ces vérités profanes sont celles d'un homme engourdi par l'alcool qui a perdu toutes ses inhibitions et qui transgresse les interdits en récitant tous les jurons qu'il connaît. Il traite le curé de "bourreau du Vatican", de "maudit instruit à'marde». Au curé qui le somme de se contenir, le juge de paix rétorque:

C'est quoi vos responsabilités à vous, hein? On sait ben, les curés vous êtes jamais responsables de rien... Vous autres, c'est le destin, la volonté de Dieu... Coudonc, Dieu là, y s'amuse tu à les faire brûler en haut? Ah non, c'est vrai, ils brûlent en enfer... Ben la p'tite, c'est ça qu'elle a vécu... Un enfer. [...] Ça, c'est de vot' faute. Tout ça c'est de vot' faute pis c'est ça que moi j'dis.

$$
++4
$$

60. MARE-STEPHANE, «Les tristes aurores", La terre de chez nous, 14 juillet 2005, p. 27.

61. Voir Gilles LABELLE, "Sens et déclin de la colère antıthéologique après la Révolution tranquille", Jacques PALLARD, Alan G. GAGNON et Bernard GAGNON [dir.], Divernité et adentités au Quebec et dans les regions d'Europe, Québecl Bruxelles, Presses de l'Université Laval/Presses universitaires européennes, 2005. 
Hautain, le curé fait valoir qu'il est un "penseur", un "intellectuel" qui n'était pas destiné à être curé dans une paroisse de cultivateurs.

Dans ce court échange entre le juge de paix et le curé, il y a tout le ressentiment d'un certain Québec moderne contre l'Église et ses infâmes représentants du clergé. Le curé Leduc, représentant de l'Église et intellectuel, est le véritable bouc émissaire de l'histoire de Luc Dionne, le paratonnerre qui absorbe toutes les frustrations de la communauté. D'une certaine façon, sa culpabilité absout la marâtre, Télesphore et les villageois, c'est-à-dire "le monde ordinaire", le "vrai monde", victime des forces sociales d'une époque heureusement révolue. "Quoi de plus rassurant que de pointer sa vengeance sur un seul? ", se demande Simon Galiero, "Quoi de plus consensuel, de plus cliché que d'affubler d'un curé infâme la collectivité québécoise du passé?" Aurore est, selon Galiero, "la définition même d'un film qui est non pas populaire, mais populiste avec ce que cela suppose de plus bas instincts ${ }^{62}$ ". Un avis partagé par le critique Martin Bilodeau, qui sent dans ce film "le cynisme et l'opportunisme d'entrepreneurs du cinéma qui, enivrés par le triomphe de Séraphin, cherchent le Klondike dans le répertoire de la Grande Noirceur ${ }^{63}$ ". La rédactrice en chef de la revue 24 Images, Marie-Claude Loiselle, est encore plus sévère:

En stigmatisant à outrance cette figure du curé qui, de surcroît, est aussi... un intellectuel [...], Luc Dionne ne fait pas que s'en prendre à l'Église et à la religion avec tout l'arsenal de clichés propres à notre époque, il se substitue lui-même à la figure du conspué. Ne réalise-t-il pas, aussi bien que tous les autres glorieux rassembleurs du petit et grand écran, qu'il est un de ces nouveaux curés, gardien d'un nouvel obscurantisme peut-être bien plus pernicieux que celui de jadis, qu'il appuie son autorité - c'est-à-dire celle que l'on confere aujourd'hui aux "gens des médias» - sur sa capacité à priver le public de toute source d'élévation, morale et intellectuelle; qui le maintient, tout comme l'élite d'hier, dans le petit, l'étroit, le bas, renforçant sans cesse davantage une image amoindrie et pitoyable de nous tous ${ }^{64}$ ?

Et Marie-Claude Loiselle de demander: «Sommes-nous vraiment ce que l'écran nous renvoie ${ }^{65}$ ?"

$$
+4
$$

62. Simon Galiero, "Aurore. Vengeance ex profit", Hors champ, octobre 2005, http://www.horschamp.qc.cal article.php3? ıd_article= 192 ( 5 décembre 2007).

63. Martin BILODEAU, "Aurore. Vie et morr d'une pop-martyre», Le Devor, 2 juillet 2005 , p. E1.

64. Marie-Claude LOISELLE, "Éditorial. Sommes-nous ce que nos écrans nous renvotent? ", 24 Images, $n^{\circ} 124$, automne 2005, hrtp://www.revue24images.com/archives.php?type=extraits\&edition=124 (5 décembre 2007).

65. Ibrd. 
Contrairement à ce qu'avait espéré Heinz Weinmann, les classiques remis au goût du jour révèlent que les Québécois ne sont pas près de se réconcilier avec la foi des ancêtres, ni même avec leur passé catholique. Ces films, surtout Séraphin et Aurore, repoussent le Mal dans un passé obscurantiste duquel les Québécois se seraient glorieusement sortis depuis la Révolution tranquille. Tel Narcisse, ils contemplent dans le miroir du passé les bienfaits de notre époque. Au lieu de revisiter ce passé, d'y accéder autrement qu'à travers le filtre d'une certaine mémoire collective lourde de ressentiment, et d'ainsi, pour le dire comme le critique Martin Bilodeau, "apprendre quelque chose de nouveau sur nous-mêmes, en tant que peuple québécois ${ }^{66} "$, ces films, fidèles aux lois du genre, se collent aux représentations dominantes. Malgré la sortie de films comme La neuvaine ou Contre toute espérance, de Bernard Émond, qui semblent beaucoup plus ouverts au langage de la transcendance et moins prompts à renier l'héritage judéochrétien qui a tant marqué l'histoire du Québec, le genre historique récent, éminemment populaire, continue de présenter le passé catholique canadienfrançais comme un vaste musée des horreurs. En 2005, La neuvaine n'a en effet attiré que 66000 spectateurs. Nous sommes très loin des 707000 spectateurs d'Aurore la même année. Comme le veut la loi du genre, on chercherait en vain, dans ces films, une prise de position politique contre le fait religieux ou des arguments contre le laïcisme. Alors que l'Église catholique est désertée, que les communautés religieuses ont beaucoup de mal à recruter et que la société se déchristianise, nous faisons plutôt face à la vieille haine d'une génération lyrique qui préfere s'en prendre à une institution exsangue plutôt que de se pencher sur ses propres angoisses spirituelles ${ }^{67}$.

Ces films, est-ce nécessaire d'insister, ne s'intéressent guère au passé, encore moins aux ancêtres qui y ont vécu. Comme le signale assez justement Marie-Claude Loiselle,

le regard que portent les Binamé, Canuel et Dionne sur ce temps révolu, embaumé, dans lequel se déroule l'action de leurs films est explicitement condescendant à l'égard du petit peuple de paysans et d'ouvriers qui, presque tous, nous apparaissent bêtas, mous et sans aucune grandeur morale [...] comme si les "metteurs en images" de

$$
+++
$$

66. Martin BILOdeAU, «Lamour dans les Pays-d'en-haut», Le Devorr, 30 novembre 2002, p. E7.

67. Voir Daniel TANGUAY, "Au chevet de l'Église catholıque québécoise. Réflexions d'un mini-boomern, Argument, vol. 8, $n^{\circ} 2$, printemps-été 2006, p. 26-37. 
ces films, captifs du monde actuel qui ne doivent rien à personne, les envisageaient (et les jugeaient) de haut ${ }^{68}$.

Cette représentation caricaturale a d'ailleurs été exploitée par des publicitaires. Le succès de Séraphin est à l'origine d'une campagne promotionnelle de Bell Canada où l'on voyait des Canadiens français d'autrefois dépeints en paysans arriérés, incultes, un peu demeurés sur les bords ${ }^{69}$. Cette posture condescendante a en effet de quoi étonner, puisque ce passé n'a au fond rien de particulièrement déshonorant si on le compare avec celui de bien d'autres nations. Ce qui étonne aussi dans cette arrogance moderniste, mais combien révélatrice des "metteurs en images", c'est que les Québécois ont longtemps fondé leur quête d'autonomie sur l'histoire, sur "notre maître le passé ${ }^{70}$ ". Aujourd'hui, ce passé semble devenu honteux ou source de "mauvaise conscience" pour le dire comme Jacques Beauchemin ${ }^{71}$. Ces films, faut-il le rappeler, sont ceux d'une génération. Il sera intéressant de voir comment la prochaine génération de cinéastes, confrontée à la dissolution du lien social, à la "dégénération ${ }^{72}$ " de l'idée de filiation, à l'exacerbation du consumérisme hédoniste et au vide de sens de nos sociétés "ultramodernes", envisagera ce passé.

68. Marie-Claude LOISELLE, op. cit.

69. Louis ROUSSEAU, a Entre la quête des possibles et celle du fondement, le travasl de la mémoire relıgieusen, E.-Martin MEUNIER et Joseph Yvon THÉRIAULT [dır.], Les impasses de la mémotre. Histoire, filiation, nation et religzon, Montréal, Fides, 2007, p. 321.

70. Pour reprendre la célèbre formule de Lionel Groulx.

71. Jacques BEAUCHEMIN, Lhistoire en trop. La matuaise conscience des souveraınistes québécots, Moncréal, VLB éditeur, 2002.

72. Tìre d'une chanson désormais célèbre du groupe Mes aïeux dont les membres ont tous environ 30 ans. 